Jean-Paul Cadet, Christophe Guitton (dir.), Les professions intermédiaires. Des métiers d'interface au cour de l'entreprise

Paris, Armand Colin, coll. « Recherches », 2013

Jérôme Deauvieau

\title{
OpenEdition
}

Journals

Édition électronique

URL : http://journals.openedition.org/travailemploi/6734

DOI : 10.4000/travailemploi.6734

ISSN : $1775-416 \mathrm{X}$

Éditeur

DARES - Ministère du Travail

Édition imprimée

Date de publication : 1 juillet 2015

Pagination : 78-79

ISSN : 0224-4365

Référence électronique

Jérôme Deauvieau, « Jean-Paul Cadet, Christophe Guitton (dir.), Les professions intermédiaires. Des métiers d'interface au cœur de l'entreprise », Travail et Emploi [En ligne], 143 | juillet-septembre 2015, mis en ligne le 01 juillet 2015, consulté le 24 septembre 2020. URL : http://journals.openedition.org/ travailemploi/6734; DOI : https://doi.org/10.4000/travailemploi.6734

Ce document a été généré automatiquement le 24 septembre 2020.

(c) Direction de l'animation de la recherche, des études et des statistiques (Dares) 


\section{Jean-Paul Cadet, Christophe Guitton (dir.), Les professions intermédiaires. Des métiers d'interface au cœur de l'entreprise}

Paris, Armand Colin, coll. « Recherches », 2013

Jérôme Deauvieau

\section{RÉFÉRENCE}

Jean-Paul Cadet, Christophe Guitton (dir.), Les professions intermédiaires. Des métiers

d'interface au cœur de l'entreprise, Paris, Armand Colin, coll. « Recherches », 2013, 393 p.

L'ouvrage collectif, Les professions intermédiaires. Des métiers d'interface au cour de l'entreprise, dirigé par Jean-Paul Cadet et Christophe Guitton, s'appuie sur les résultats d'un programme de recherche pluriannuel du Centre d'études et de recherches sur les qualifications (Céreq), réalisé entre 2008 et 2012. C'est peu dire que ce travail vient remplir un vide dans la recherche en sciences sociales, tant les professions intermédiaires constituent, selon les termes des auteurs, "un point aveugle des sciences humaines et sociales " (p. 20). L'ouvrage s'attache à décrire une région du salariat peu étudiée, qui plus est avec l'ambition d'en saisir les caractéristiques générales et les évolutions. Il est centré sur les professions intermédiaires en entreprise et s'intéresse donc principalement aux contremaîtres et agents de maîtrise, aux techniciens, et aux professions intermédiaires administratives et commerciales des entreprises. Il étudie dans une moindre mesure la catégorie des professions intermédiaires de la santé et du travail social, mais laisse logiquement de côté celle des professions intermédiaires de la fonction publique ainsi que celle des instituteurs et assimilés. L'ensemble analysé n'en demeure pas moins numériquement important puisqu'il concentre près du quart de l'emploi salarié du privé. Les moyens et les modes 
d'investigations mobilisés pour mener à bien l'étude impressionnent : pas moins d'une trentaine de chercheurs, pour la plupart rattachés au Céreq, un travail de plusieurs années qui a conjugué monographies d'entreprises et de professions (soixante-cinq métiers étudiés dans le programme de recherche), des analyses secondaires d'enquêtes de la statistique publique et des revues de la littérature.

Ce dispositif met les auteurs en capacité de répondre à un large questionnement qui se décline en trois niveaux. Le premier niveau étudie le contenu des activités de travail et la position des professions intermédiaires dans l'entreprise. Il vise ainsi à saisir la nature des activités et des responsabilités exercées, le degré de spécialisation du travail et son partage entre technicité et management. Il cherche également à examiner le rôle et la place des professions intermédiaires dans l'organisation du travail, en les comparant avec les cadres d'un côté et les ouvriers et employés de l'autre. Le deuxième niveau de questionnement s'intéresse à la formation et à la professionnalisation, à travers l'étude du rôle de la formation continue pour ces professions qui en sont les principaux bénéficiaires dans les entreprises. Enfin, le dernier niveau a trait à l'emploi et aux carrières, et tente de comprendre à la fois les modes d'alimentation des emplois de professions intermédiaires et leurs destinées socioprofessionnelles. La conjugaison de ces deux perspectives permet de s'interroger sur la place des professions intermédiaires dans les espaces de carrières socioprofessionnelles en entreprise.

3 L'ouvrage est construit en trois parties, encadrées par un avant-propos et une conclusion de José Rose donnant à voir le contexte de réalisation de l'étude et ses enseignements principaux.

4 La première partie est consacrée à une description des professions intermédiaires en entreprise, qui permet dans le même temps de dégager les lignes de questionnement. Les professions intermédiaires y sont d'abord saisies d'un point de vue statistique (chapitre 1), notamment en distinguant les évolutions des professions intermédiaires administratives et commerciales des entreprises, des techniciens et des contremaitres. L'attention est ensuite portée sur l'insertion des sortants de formation initiale de niveau III au sein des professions intermédiaires (chapitre 2). Les trois chapitres suivants sont l'occasion d'un travail bibliographique sur les professions intermédiaires en entreprise (chapitre 3), l'encadrement de proximité (chapitre 4) et les professions intermédiaires de l'éducation, de la santé et du travail social (chapitre 5).

La deuxième partie de l'ouvrage est constituée d'un exposé de quinze monographies de professions intermédiaires d'entreprise, regroupées par leur fonction principale. Sont ainsi différenciés les métiers à dominante managériale, à dominante technique, à dominante commerciale puis administrative et gestionnaire. Malgré ce judicieux mode d'exposition des monographies, on n'évite pas ici une certaine hétérogénéité dans les présentations. Soulignons cependant qu'il est très rare de disposer d'un tel éventail de professions d'un même niveau de qualification, permettant de toucher du doigt la grande diversité de situations mais également de mettre en évidence certains processus transversaux qui sont analysés dans la dernière partie.

6 Le premier chapitre de la troisième partie tente en effet de relier les monographies entre elles et de dégager certains mécanismes à l'œuvre au sein des professions intermédiaires des entreprises, et même au-delà dans le salariat (chapitre 21). En traitant de manière systématique les soixante-cinq métiers étudiés dans le programme de recherche, Christophe Guitton met en évidence deux éléments décisifs des transformations et des tensions du travail salarié des professions intermédiaires: la 
montée de la polyvalence fonctionnelle des emplois, avec une interpénétration des dimensions managériale, technique, commerciale et administrative; et le fait que les professions intermédiaires sont au cœur d'un « processus multiforme de rationalisation du travail et de managérialisation de l'activité». Ces nouvelles exigences professionnelles expliquent en grande partie pourquoi la formation continue est majoritairement concentrée sur les professions intermédiaires en entreprise (chapitre 25).

7 Cette troisième partie est également l'occasion de revenir sur la façon dont les professions intermédiaires s'insèrent dans les espaces de carrières en entreprise. Le chapitre 24 s'intéresse ainsi au recrutement des professions intermédiaires, et rend compte des déterminants des modes d'alimentation de ces professions, entre recrutement par promotion interne ou par embauche de jeunes entrants sur le marché du travail. Ce questionnement est poursuivi dans le chapitre 26, mais cette fois-ci sous l'angle de la destinée, en cherchant à comprendre pourquoi les entreprises continuent à promouvoir des professions intermédiaires au statut cadre, et complété par un dernier chapitre visant à expliquer les réticences d'une partie des professions intermédiaires pour une promotion au statut cadre.

On referme cet ouvrage en ayant beaucoup appris sur une région méconnue du salariat. Qu'il s'agisse des enjeux autour des métiers des professions intermédiaires, de la question de la formation et de la professionnalisation, ou de la place des professions intermédiaires dans les espaces de carrières socioprofessionnelles, le panorama proposé est vaste. Ajoutons à cela les grandes qualités formelles de l'ouvrage, tant du point de vue de la construction des parties que de la clarté du propos. Pour toutes ces raisons, nous sommes clairement ici en présence de la publication de référence sur le sujet.

9 Sa lecture permet également plus largement d'alimenter la réflexion sur les dynamiques récentes du salariat, et sera notamment très utile pour tout utilisateur de la nomenclature des professions et catégories socioprofessionnelles de l'Insee ${ }^{1}$. Mieux comprendre qui sont les professions intermédiaires d'entreprise, c'est en effet saisir du même coup ce qui les distingue des catégories connexes, tout particulièrement les cadres et les employés, et par là même mieux comprendre la logique d'ensemble de cette nomenclature si précieuse pour étudier la structure socioprofessionnelle française. Il ne reste plus qu'à souhaiter que ce type de programmes de recherche, qui ne peuvent être mis en œuvre que dans des institutions telles que le Céreq, puisse être renouvelé pour aboutir à d'autres publications du même ordre, par exemple sur les professions intermédiaires du secteur public.

\section{NOTES}

1. Institut national de la statistique et des études économiques. 


\section{AUTEURS}

\section{JÉRÔME DEAUVIEAU}

École normale supérieure, Centre Maurice Halbwachs (UMR 8097, CNRS / EHESS / ENS) 